APS

physics

This is the accepted manuscript made available via CHORUS. The article has been published as:

\title{
Sub-shell closure and shape coexistence in the transitional nucleus $\wedge\{98\} \mathrm{Zr}$
}

W. Witt, V. Werner, N. Pietralla, M. Albers, A. D. Ayangeakaa, B. Bucher, M. P. Carpenter, D. Cline, H. M. David, A. Hayes, C. Hoffman, R. V. F. Janssens, B. P. Kay, F. G. Kondev, W. Korten, T. Lauritsen, O. Möller, G. Rainovski, G. Savard, D. Seweryniak, J. Smith, R.

Stegmann, S. Zhu, and C. Y. Wu

Phys. Rev. C 98, 041302 - Published 16 October 2018

DOI: 10.1103/PhysRevC.98.041302 


\title{
Sub-Shell Closure and Shape Coexistence in the transitional nucleus ${ }^{98} \mathrm{Zr}$
}

\author{
W. Witt ${ }^{1}$, V. Werner ${ }^{1}$, N. Pietralla ${ }^{1}$, M. Albers ${ }^{2}$, A.D. Ayangeakaa ${ }^{2,10}$, B. Bucher ${ }^{4}$, M.P. Carpenter ${ }^{2}$, D. Cline ${ }^{3}$, \\ H.M. David ${ }^{8}$, A. Hayes ${ }^{11}$, C. Hoffman ${ }^{2}$, R.V.F. Janssens ${ }^{2,6,7}$, B.P. Kay ${ }^{2}$, F.G. Kondev², W. Korten ${ }^{9}$, T. Lauritsen ${ }^{2}$, \\ O. Möller ${ }^{1}$, G. Rainovski ${ }^{5}$, G. Savard², D. Seweryniak ${ }^{2}$, J. Smith ${ }^{2}$, R. Stegmann ${ }^{1}$, S. Zhu ${ }^{2}$, C.Y. Wu ${ }^{4}$ \\ ${ }^{1}$ Institut für Kernphysik, Technische Universität Darmstadt, 64289 Darmstadt, Germany \\ ${ }^{2}$ Argonne National Laboratory, Argonne, IL 60439, USA \\ ${ }^{3}$ Department of Physics and Astronomy, University of Rochester, Rochester, NY 14627, USA \\ ${ }^{4}$ Lawrence Livermore National Laboratory, Livermore, CA 94550, USA \\ ${ }^{5}$ Faculty of Physics, St. Kliment Ohridski University of Sofia, 1164 Sofia, Bulgaria \\ ${ }^{6}$ Department of Physics and Astronomy, University of North Carolina, NC 27599, USA \\ ${ }^{7}$ Triangle Universities Nuclear Laboratory, Duke University, NC 27708, USA \\ ${ }^{8}$ GSI Helmholtzzentrum für Schwerionenforschung, 64291 Darmstadt, Germany \\ ${ }^{9}$ Irfu, CEA, Université Paris-Saclay, F-91191 Gif-sur-Yvette, France \\ ${ }^{10}$ Department of Physics, United States Naval Academy, Annapolis, MD 21402, USA and \\ ${ }^{11}$ Institute of Optics, University of Rochester, Rochester, NY 14642, USA
}

(Dated: August 5, 2018)

\begin{abstract}
In the rapid shape change from spherical to deformed nuclei in the $Z=40 \mathrm{Zr}$ isotopic chain, recent work has identified shape coexistence in ${ }^{96} \mathrm{Zr}$. Between ${ }^{96} \mathrm{Zr}$ and the strongly-deformed ${ }^{100} \mathrm{Zr},{ }^{98} \mathrm{Zr}$ is expected to also exhibit coexistence of nuclear shapes. The degree of mixing between different configurations is mainly determined by the nucleon-nucleon interactions. For nuclear model predictions, experimental constraints are needed, but barely available for ${ }^{98} \mathrm{Zr}$. To study low-lying transitions in ${ }^{98} \mathrm{Zr}$, a Coulomb excitation experiment was conducted at the ATLAS facility at ANL using a ${ }^{98} \mathrm{Zr}$ beam extracted from the CARIBU ion source and GRETINA for $\gamma$-ray spectroscopy coupled to CHICO2 for ion detection. This paper reports on the first decisive deduction of the $B\left(E 2 ; 2_{1}^{+} \rightarrow 0_{1}^{+}\right)$transition strength in ${ }^{98} \mathrm{Zr}$ and on its interpretation.
\end{abstract}

The emergence of collective behavior out of the singleparticle motion of the valence nucleons is the basis for understanding how nuclear structure evolves with the number of valence protons and neutrons. First ideas of nuclear deformation as direct effect of neutron-proton correlations, eventually resulting in the early filling of higher orbitals, have already been discussed since the late 70's [1-3]. By now, these effects are well known and commonly accepted to lead, for example, to the occurrence of so-called islands of inversion through the tensor force. This occurs, e.g., in the region of neutron-rich $\mathrm{Na}$ and $\mathrm{Mg}$ isotopes [4-7], where the traditional $N=20$ neutron shell gap vanishes, or in neutron-rich isotopes below $Z=28$ [8,9], where quadrupole correlations support an early filling of higher-lying orbits beyond the "normal" valence space. When certain configurations, which would be suppressed in a normal ordering of shell-model orbitals, become energetically favorable, shape coexistence can result; i.e., the presence of different deformations either mixed into the eigenstates of the nucleus, or of different eigenstates characterized by distinct nuclear deformations. Already the addition of only two nucleons to an even-even nucleus can change the nucleon configurations drastically. This is manifested by the occurrence of shape-phase transitions [10] such as; e.g., in $N=90$ isotopes [11-13]. Besides this type-I evolution of collectivity, Togashi et al. [14] have recently pointed out another mechanism named type-II shell evolution. In the latter, the reconfiguration of nucleons within a given nucleus, along the lines of Ref. [1-3], can lead to changes in the effective shell-model structure as a function of ex- citation energy, similar to the impact brought about by a changing number of nucleons.

The Zr isotopes have been identified as a good example for type-II shell evolution, strongly supported by recent electron-scattering experiments on ${ }^{96} \mathrm{Zr}$ [15] (and reanalysis within Ref. [16]). The ground state and the $2_{1}^{+}$level were found to be nearly spherical from the $2_{1}^{+}$excitation energy and the small $B(E 2)$ transition strength of $2.3(3)$ W.u. connecting it to the $0_{1}^{+}$ground state. In contrast, the first excited $0_{2}^{+}$level and the quadrupole-excited $2_{2}^{+}$ state located on top of it were found to be associated with large deformation based on a large $B\left(E 2 ; 2_{2}^{+} \rightarrow 0_{2}^{+}\right)$value of 36(11) W.u. Similar observations have previously been reported for ${ }^{94} \mathrm{Zr}$ [17]. A distinction between shape coexistence in ${ }^{96} \mathrm{Zr}$ and others found in the nuclear chart was found $[15,16]$. The formation of a deformed excited structure in the Zr isotopes, in the presence of a spherical ground state up to $N=58$, has been predicted by state-of-the-art Monte Carlo Shell Model (MCSM) calculations [14], but is backed by transition strength data only up to $N=56$. From the systematics of Fig. 1, a significant rise in the $R_{4 / 2}=\frac{E\left(4_{1}^{+}\right)}{E\left(2_{1}^{+}\right)}$ratio between ${ }^{96} \mathrm{Zr}$ and ${ }^{100} \mathrm{Zr}$ is clearly visible. This rise is accompanied by an increase in the $B\left(E 2 ; 2_{1}^{+} \rightarrow 0_{1}^{+}\right)$value from $2.3(3)$ W.u. to 80.5(44) W.u. between these two isotopes, while their respective $2_{1}^{+}$energies decrease from 1751 to $213 \mathrm{keV}$. This change in the ground-state structure is most likely due to the presence of the excited deformed band, as in ${ }^{96} \mathrm{Zr}$, which becomes energetically favored and, hence, evolves into the ground state of ${ }^{100} \mathrm{Zr}$.

In general, in a shape-phase transition of first order 


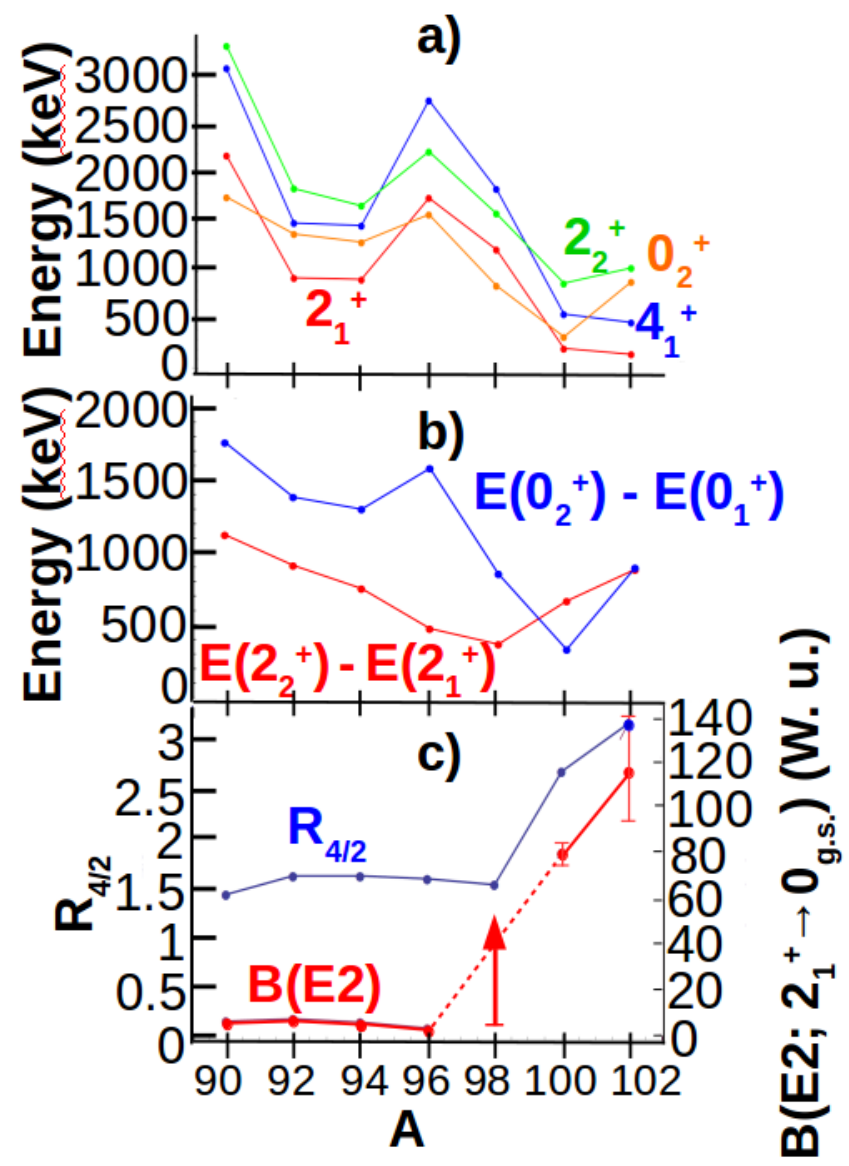

FIG. 1. Systematics of observables in the Zr isotopic chain: a) Development of the low-lying level energies, b) energy differences of $2^{+}$and $0^{+}$states and c) $R_{4 / 2}$ (blue, left-hand scale) and $B(E 2)$ (red, right-hand scale) values of the ground states indicating a shape transition. Only a lower limit was available for the $B(E 2)$ value for ${ }^{98} \mathrm{Zr}$ prior to this work (shown by the arrow) and, hence, the (dashed) line linking ${ }^{96} \mathrm{Zr}$ and ${ }^{100} \mathrm{Zr}$ is simply to guide the eye.

the two unperturbed structures, here the spherical and the deformed one, exchange in energy. Since interactions between both structures will be finite, even if small, the mixing between states with the same angular momentum will lead to an avoided crossing; i.e., these states will exchange character, but will not be degenerate. This behavior can be seen in Fig. 1 (b) showing the difference of the energies of the lowest $0^{+}$and $2^{+}$states through the $\mathrm{Zr}$ isotopic chain. The $0_{1}^{+}$and $0_{2}^{+}$levels are closest in ${ }^{100} \mathrm{Zr}$, marking the isotope nearest to the crossing of both structures, which is the point where both unperturbed structures should be maximally mixed. Assuming that the $2_{1}^{+}$and $2_{2}^{+}$states up to ${ }^{96} \mathrm{Zr}$ correspond to the (nearly) unperturbed spherical and deformed configurations, respectively, a similar picture should occur. One observes that the closest approach of both states occurs already in ${ }^{98} \mathrm{Zr}$, which suggests that the $2^{+}$states exchange their characters earlier than their respective $0^{+}$ states. Given that the level spacings in the deformed structure are smaller than in the spherical one, this behavior can, in fact, be expected.

These considerations make ${ }^{98} \mathrm{Zr}$ a key isotope in this isotopic chain. The questions to be addressed are whether the spherical configuration is still dominant in the ground state, and how the phase transition affects the structures of the excited states. The significant E0 strength of $\rho^{2}\left(E 0 ; 0_{2}^{+} \rightarrow 0_{1}^{+}\right)$points to the necessity of taking mixing into account. However, this may also result from a large deformation difference between both states. Hence, information on $B(E 2)$ values of ${ }^{98} \mathrm{Zr}$ represents a sensitive test of the available theoretical descriptions, not only of the $\mathrm{Zr}$ isotopes, but for this entire region.

Experimental information on the $B\left(E 2 ; 2_{1}^{+} \rightarrow 0_{1}^{+}\right)$ value in ${ }^{98} \mathrm{Zr}$, which would be capable of deciding between a deformed-collective or a weakly-collective vibrational structure, is scarce to date. The only available data give a lower bound of 1.83 W.u. obtained from a fast-timing lifetime measurement [18]. Here, we present the first measurement of a decisive upper bound to this $B(E 2)$ value from a Coulomb excitation experiment performed with a radioactive ${ }^{98} \mathrm{Zr}$ beam. The new data indicate that the $2_{1}^{+} \rightarrow 0_{1}^{+} E 2$ transition in ${ }^{98} \mathrm{Zr}$ is not due to the rotation of a strongly quadrupole-deformed nucleus.

The experiment was performed at the ATLAS facility at Argonne National Laboratory (ANL). ${ }^{98} \mathrm{Zr}$ ions, among other nuclei, were extracted from the CARIBU source [19-21] after spontaneous fission of a $37 \mathrm{GBq}$ (1 Ci) ${ }^{252} \mathrm{Cf}$ source. The fission fragments were slowed down in a degrader foil and were subsequently thermalized with high efficiency and turned into a $1^{+}$charged-ion beam by a gas catcher. After a first mass selection by the CARIBU isobar separator, the extracted ions were transferred to an ECR ion source for charge breeding. They were then injected into the ATLAS superconducting linear accelerator. Further mass separation of $A=98 \mathrm{nu}-$ clei was performed during post-acceleration by magnetic rigidity selection. Nevertheless, the isobars of ${ }^{98} \mathrm{Zr}$ from Sr to Mo were the main components of the final beam "cocktail". The beam composition was analyzed using a $49.7 \mathrm{mg} / \mathrm{cm}^{2}{ }^{n a t} \mathrm{Au}$ target stopping the beam about $2 \mathrm{~m}$ downstream from the reaction target. A single Ge detector with a detection efficiency of $\epsilon_{\gamma}=0.2 \%$ at $1 \mathrm{MeV}$ was placed at the beam stop. The beam-on-target time summed up to 6 days.

The beam, with an energy of $464 \mathrm{MeV}$, impinged on a $1.59 \mathrm{mg} / \mathrm{cm}^{2}$ thick ${ }^{196} \mathrm{Pt}$ foil for Coulomb excitation of target and beam ions. The recoiling particles were detected and identified with the forward shell of CHICO2, a segmented PPAC array with a scattering angle acceptance of $12^{\circ} \leq \theta \leq 85^{\circ}$, an angular resolution of $\Delta \theta=1^{\circ}$, and a time resolution of $\Delta t \leq 500 \mathrm{ps}$ [22]. The experimental conditions ensured that only so-called safe Coulomb excitation [23] events were recorded. The deexcitation $\gamma$ rays were detected with GRETINA, a $\gamma$-ray spectrometer consisting of electrically segmented highpurity germanium crystals covering a solid angle of $1 \pi$ with a detection efficiency of $\epsilon_{\gamma}=6.5 \%$ and an intrinsic 


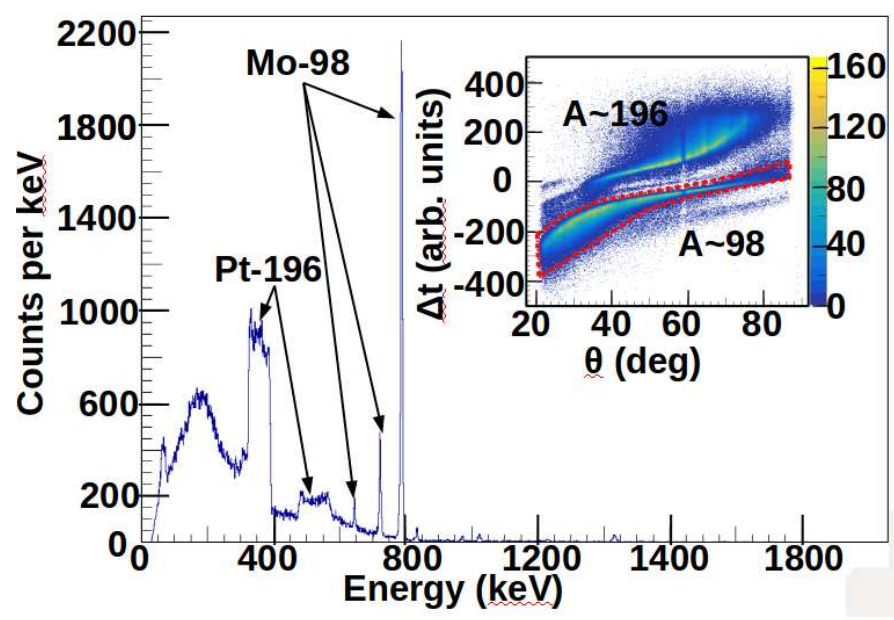

FIG. 2. Spectrum from GRETINA summed over all detector angles following Doppler correction for $A=98$ reaction products. The insert provides the difference in time of flight between the beam and the target nuclei over the scattering angle $\theta$. The selection of the $A=98$ ions is indicated by the dashed red line.

energy resolution of $\frac{\Delta E}{E} \leq 1 \%$ at $1.3 \mathrm{MeV}$ [24]. The $\gamma$ ray hit with the largest energy deposition was assumed to be the first interaction point of an event. The energy and efficiency of GRETINA and the focal plane detector were calibrated using the standard $\mathrm{Eu}, \mathrm{Ta}, \mathrm{Cs}$ and $\mathrm{Co}$ sources.

Using CHICO2, beam- $(A=98)$ and target-like $(A=$ 196) ions in the outgoing channel were separated, as illustrated in Fig. 2. Hence, outgoing ion velocity vectors were reconstructed and these were used for the Doppler correction of detected $\gamma$ rays, which were emitted in flight at velocities of about $\frac{v}{c}=8 \%$. Furthermore, the 2 mm position resolution of GRETINA was used for the Doppler correction. The powerful combination of both systems enabled an energy resolution of about $7 \mathrm{keV}$ at $800 \mathrm{keV}$. Fig. 2 provides a sum spectrum obtained with GRETINA following the application of the Doppler shift correction, in coincidence with $A=98$ events recorded in CHICO2. Shown in Fig. 3 is a zoom into the energy range of the expected $2_{1}^{+} \rightarrow 0_{1}^{+}$transition. The latter spectrum is dominated by the $3_{1}^{-} \rightarrow 2_{1}^{+}$transition of ${ }^{98} \mathrm{Mo}$ at $1230 \mathrm{keV}$. No peak corresponding to the $2_{1}^{+} \rightarrow 0_{1}^{+}$transition of ${ }^{98} \mathrm{Zr}$ is visible at $1223 \mathrm{keV}$, which should appear on the left tail of the contaminant peak from ${ }^{98}$ Mo. Nevertheless, an upper limit for the intensity of a peak at $1223 \mathrm{keV}$ with a $3 \sigma$ significance limit could be extracted.

For the deduction of this upper limit of the $2_{1}^{+} \rightarrow 0_{1}^{+}$ intensity, it was required that the intensity of a hypothetical peak at the $1223 \mathrm{keV}$ nominal energy has a $3 \sigma$ - significance, considering the maximum possible values of the peaks' FWHM, as determined from fits to nearby transitions in ${ }^{98} \mathrm{Mo}$. A linear background was used and its, as well as the energy calibration's, minor uncertain- ties are accounted for by the fit. The procedure is illustrated in Fig. 3, showing a significant $1223-\mathrm{keV}$ peak on the tail of the ${ }^{98} \mathrm{Mo} 1230-\mathrm{keV}$ transition for the best value of the FWHM, and for a maximum possible FWHM, resulting in an upper limit of 40 counts for the $2_{1}^{+} \rightarrow 0_{1}^{+}$ transition in ${ }^{98} \mathrm{Zr}$.

The amount of ${ }^{98} \mathrm{Zr}$ in the beam was extracted by decay spectroscopy of the components at the beam stop. For this, well-known level schemes, $\gamma$ - and $\beta$ decay branching ratios $[25,26]$ of ${ }^{98} \mathrm{Sr},{ }^{98} \mathrm{Y},{ }^{98} \mathrm{Nb}$ and (if existing) their isomers, and the overall decay chain $\mathrm{Sr} \rightarrow \mathrm{Y} \rightarrow \mathrm{Zr} \rightarrow \mathrm{Nb} \rightarrow \mathrm{Mo}$ were employed. From the intensities of observed $\gamma$-ray transitions in ${ }^{98} \mathrm{Y},{ }^{98} \mathrm{Zr}$ and ${ }^{98} \mathrm{Mo}$, rates of ${ }^{98} \mathrm{Sr}[113(9) \mathrm{pps}],{ }^{98} \mathrm{Y}[159(23) \mathrm{pps}]$ and ${ }^{98} \mathrm{Y}^{m}$ [101(20) pps], and the sum of ${ }^{98} \mathrm{Nb},{ }^{98} \mathrm{Nb}^{m}$ and ${ }^{98} \mathrm{Zr}$ [218(44) pps] were deduced. Less than 5 pps of ${ }^{98} \mathrm{Nb}^{m}$ could be identified in the beam. Following the independent $\mathrm{Cf}$ fission yield division of isomeric state to total amount of ${ }^{98} \mathrm{Nb}$ of 0.15 [27], <35 pps can be attributed to ${ }^{98} \mathrm{Nb}$ and its isomer, leading to a ${ }^{98} \mathrm{Zr}$ rate of at least 162 pps. The intensity ratios of this deduced beam composition are in agreement with the CARIBU yield estimates [28].

All $A=98$ projectiles contributed to the Coulomb excitation of the ${ }^{196} \mathrm{Pt}$ target. The corresponding crosssections and expected yields for low-lying transitions of the involved nuclei were calculated with the computer code CLX by H. Ower et al. [29] and compared to the data. Neglecting minor $Z$-dependent differences, the unstable $A=98$ nuclei were assumed to contribute to the target excitations according to their relative beam intensities. The surplus of target excitations, which cannot be attributed to other beam components (Nb, Mo, Y, Sr) must be attributed to ${ }^{98} \mathrm{Zr}$.

From the beam composition, the obtained yield of target excitation and the upper limit on the intensity of the $2_{1}^{+} \rightarrow 0_{1}^{+}$transition of ${ }^{98} \mathrm{Zr}$, an upper limit for the $B\left(E 2 ; 2_{1}^{+} \rightarrow 0_{1}^{+}\right)$value of ${ }^{98} \mathrm{Zr}$ was obtained. The limiting value of 40 counts for the transition of interest would translate into a value of $8.9(20) \mathrm{W} . u$. for the $B\left(E 2 ; 2_{1}^{+} \rightarrow 0_{1}^{+}\right)$strength of ${ }^{98} \mathrm{Zr}$, with the uncertainty stemming essentially from the beam composition. Hence, a conservative (definite) upper limit of 11 W.u. is deduced from the present work.

With the lower limit on the $B(E 2)$ value from a previous measurement [18], the $B(E 2)$ value of ${ }^{98} \mathrm{Zr}$ is herewith constrained to 1.83 W.u. $<B\left(E 2 ; 2_{1}^{+} \rightarrow 0_{1}^{+}\right)<11$ W.u.

The newly constrained $B(E 2)$ value of ${ }^{98} \mathrm{Zr}$ is included in the updated systematics of the $\mathrm{Zr}$ chain found in Fig. 4. Its relatively low value with an upper bound of $11 \mathrm{~W}$.u. and a lower one near 2 W.u. compares well to the $B(E 2)$ values of ${ }^{92,94} \mathrm{Zr}$ of $\sim 6 \mathrm{~W}$.u., or $2 \mathrm{~W} . u$. for ${ }^{96} \mathrm{Zr}$, reflecting a rather small deformation or the absence of collectivity in the ground state. In contrast, the next even-even isotope, ${ }^{100} \mathrm{Zr}$, is highly collective, hence, deformed in its ground state, with a $B(E 2)$ value of $80.5(44)$ W.u. In the following, these findings are discussed with respect 


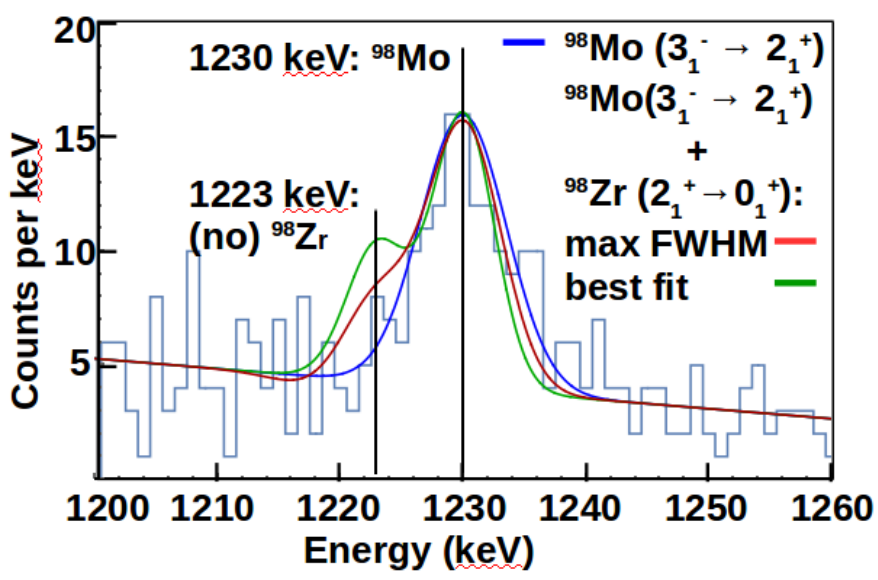

FIG. 3. Doppler-corrected energy spectrum obtained with GRETINA summed over all detector angles. Shown in blue (lowest line at $1223 \mathrm{keV}$ ) is a fit to solely the $3_{1}^{-} \rightarrow 2_{1}^{+}$transition peak of ${ }^{98} \mathrm{Mo}$ at $1230 \mathrm{keV}$, in red (middle line at 1223 $\mathrm{keV}$ ) and green (upper line at $1223 \mathrm{keV}$ ) hypothetical contributions of the $2_{1}^{+} \rightarrow 0_{1}^{+}$transition of ${ }^{98} \mathrm{Zr}$ at $1223 \mathrm{keV}$ with 40 observed transition counts at the maximum possible FWHM (red, middle line) and best fitted value of the FWHM (green, upper line). See text for further details.

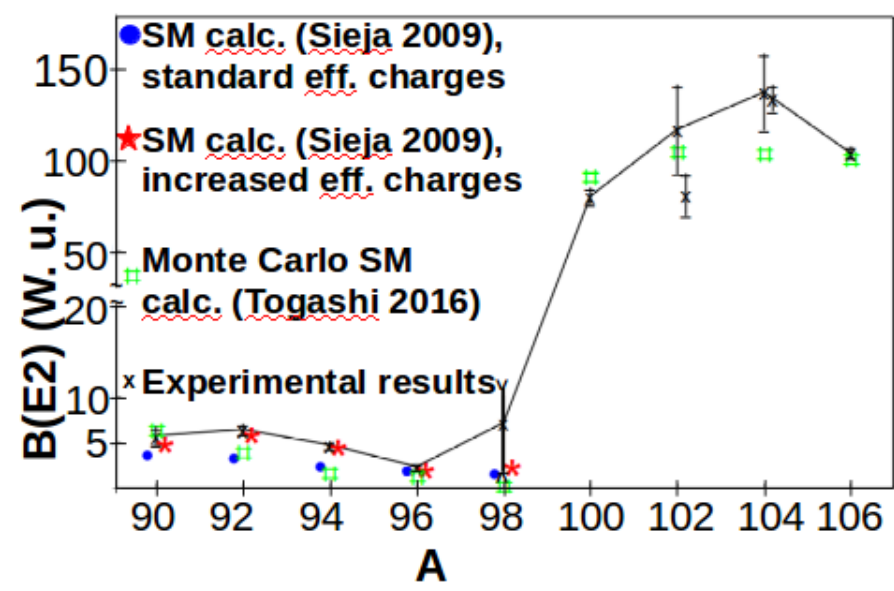

FIG. 4. Experimental $B\left(E 2 ; 2_{1}^{+} \rightarrow 0_{1}^{+}\right)$values in the $\mathrm{Zr}$ chain and shell-model calculations (Sieja 2009 [30]) with standard $\left(\mathrm{e}_{e f f}^{\nu}=0.5, \mathrm{e}_{e f f}^{\pi}=1.5\right.$, shown by circles) and increased $\left(\mathrm{e}_{e f f}^{\nu}=0.8, \mathrm{e}_{e f f}^{\pi}=1.8\right.$, shown by stars $)$ effective charges and recent Monte Carlo shell-model calculations (Togashi 2016 [14], shown by squares). The $B(E 2)$ value of ${ }^{98} \mathrm{Zr}$ is limited by previous work [18] (lower limit) and an upper limit was established in this work. Note the change of vertical scale above 20 W.u.

to a simple shell-model frame, as well as in the context of available large-scale shell-model calculations.

In a simple picture, referring to the sequence of active orbitals, neutrons in the ground state of the $N=52-56$ $\mathrm{Zr}$ isotopes are mainly bound to the $\nu 2 d_{5 / 2}$ orbital. This is corroborated, not only by the systematics of Fig. 1, but also by the small (even negative) $2_{1}^{+} \mathrm{g}$ factors in these isotopes [31]. With $\mathrm{Zr}$ isotopes located on the $Z=40$ pro- ton sub-shell closure, which separates the $p f$ shell from the $1 g_{9 / 2}$ orbital, ${ }^{96} \mathrm{Zr}$ is located on sub-shell closures of both protons and neutrons. In a normal filling sequence of the neutron orbitals, the $\nu 3 s_{1 / 2}$ state would be filled in ${ }^{98} \mathrm{Zr}$, herewith forming another sub shell. This would account for the high $2_{1}^{+}$energies in ${ }^{96} \mathrm{Zr}$ and ${ }^{98} \mathrm{Zr}$ since, for the formation of a $2^{+}$state, in either case neutrons need to be promoted to the higher-lying $\nu 1 g_{7 / 2}$, $\nu 2 d_{3 / 2}$, or $\nu 1 h_{11 / 2}$ orbitals, and protons must overcome the $p f-g_{9 / 2}$ shell gap. The present result, reflecting low collectivity, indeed hints at a mostly inert ${ }^{98} \mathrm{Zr}$ ground state with neutrons filling the $d_{5 / 2}$ and $s_{1 / 2}$ orbitals and protons filling the $p f$ shell.

This interpretation agrees well with results of the largescale shell-model calculations by Togashi et al. [14]. These indicate that the filling of the neutron orbitals above the $s_{1 / 2}$ state is responsible for protons being promoted to the $g_{9 / 2}$ orbital, and vice versa. This occurs by excitation of either type of nucleons to their respective orbitals, leading to a coexisting deformed structure, combined with the natural population of neutron orbitals beyond the $s d$ states above $N=58$. The interplay between both mechanisms leads to the shape-phase transition in the $\mathrm{Zr}$ isotopic chain, which takes place between $N=58$ and $N=60$. Extensive theoretical studies within the IBM and the mean-field approach [32, 33] describe the shape evolution in the whole mass region reasonably well and point out the uniquely pronounced, fast structural change of $\mathrm{Zr}$ isotopes.

Included in Fig. 4 are $B(E 2)$ strengths from a shellmodel calculation [30] using standard and increased effective charges, as is often found in this region [34, 35]. The agreement between data and calculations with the increased effective charges is satisfactory up to $N=58$, but calculations for higher neutron numbers were not possible. Also included in Fig. 4 are the recent results from Ref. [14], which extend beyond $N=58$. Due to the use of a larger valence space, including in addition the proton $1 g_{7 / 2}, 2 d_{5 / 2,3 / 2}$, and $3 s_{1 / 2}$ orbitals beyond $Z=50$ and the neutron $2 f_{7 / 2}$ and $3 p_{3 / 2}$ orbitals below $N=50$, the MCSM calculation based on the JUN45 and SNBG3 interactions assumed smaller effective charges. These calculations predict small $B(E 2)$ excitation strengths up to $N=58$ with a transition to large strength at $N=60$ and beyond.

The measurement of an upper limit for the excitation yield of the $2_{1}^{+}$state in ${ }^{98} \mathrm{Zr}$ does not only limit the $B\left(E 2 ; 2_{1}^{+} \rightarrow 0_{1}^{+}\right)$transition strength, but also the value of $B\left(E 2 ; 2_{1}^{+} \rightarrow 0_{2}^{+}\right)$, since the relative intensity between both transitions is known [25]. Along with the lower bound from the direct lifetime measurement of Ref. [18], the latter is constrained to 11.5 W.u. $\leq B\left(E 2 ; 2_{1}^{+} \rightarrow\right.$ $\left.0_{2}^{+}\right) \leq 71.3 \mathrm{~W}$.u. The large-scale shell model [14] predicts a value of $\sim 70$ W.u., which is near the upper limit of the experimental range. The $B(E 2)$ value for the decay to the $0_{2}^{+}$state, which is the first excited state in the case of ${ }^{98} \mathrm{Zr}$, is about an order of magnitude more collective than that to the spherical ground state. Therefore, one can 
conclude that the $2_{1}^{+}$state is a collective excitation built on the excited $0_{2}^{+}$state rather than a spherical, weaklycollective excitation based on the ground state. The predicted strength of $70 \mathrm{~W} . u$. is close to the $2_{1}^{+}$excitation strength of $80.5(44)$ W.u. in the neighboring ${ }^{100} \mathrm{Zr}$, where both the $0_{1}^{+}$and $2_{1}^{+}$states belong to a deformed configuration. In fact, the $2_{2}^{+}$state follows closely the evolution of the $0_{2}^{+}$level up to $A=98$, where its energy is closest to that of the $2_{1}^{+}$state, indicating a switch of the two configurations (see Fig. 1). The $0_{1}^{+}$and $0_{2}^{+}$states are closest at $A=100$. Clearly, a higher precision of both $B(E 2)$ values in ${ }^{98} \mathrm{Zr}$ is required to quantify further the actual collectivity of the quadrupole excitation of the excited $0^{+}$ state, and to draw conclusions on the degree of mixing between spherical and deformed configurations into the $2_{1}^{+}$level. In the event that a value close to the predicted 70 W.u. for the $2_{1}^{+} \rightarrow 0_{2}^{+}$transition would turn out to be correct, the corresponding reduced ground-state transition strength would be on the order of 10 W.u., representing a somewhat increased collectivity in the decay to the $0_{1}^{+}$state as compared to the values of 2-6 W.u. seen in the lighter $\mathrm{Zr}$ isotopes. This would indicate a non-negligible, $\approx 20 \%$, mixing of spherical and deformed configurations in ${ }^{98} \mathrm{Zr}$. Hence, the accurate measurement of the expected large $B\left(E 2 ; 2_{1}^{+} \rightarrow 0_{2}^{+}\right)$transition strength will serve as a sensitive measure of the barrier between spherical and deformed structure, and as a stringent test for state-of-the-art nuclear models and their ability to account for the subtle mechanisms responsible for structure evolution in heavy nuclei.

To conclude, for the first time, upper bounds for the $B(E 2)$ values of the transitions from the $2_{1}^{+}$level of ${ }^{98} \mathrm{Zr}$ to the ground state and to the first excited $0_{2}^{+}$state have been obtained through the combination of the GRETINA and CHICO2 devices while taking advantage of the availability of a ${ }^{98} \mathrm{Zr}$ radioactive beam at the ATLAS facility. The new data favor a weakly (if at all) collective excitation from the ground state, but a comparably strong excitation from the first excited $0_{2}^{+}$state, in agreement with recent state-of-the-art calculations. The results indicate that ${ }^{98} \mathrm{Zr}$ is near to the shape-phase transition between spherical and deformed ground states in the $\mathrm{Zr}$ isotopic chain. The one order of magnitude difference in the $B(E 2)$ decay strengths of the $2_{1}^{+}$state to both lowlying $0^{+}$states is indicative of the large difference in the structures of the two $0^{+}$states. However, a conclusion on the degree of mixing of spherical and deformed configurations into the $2_{1}^{+}$state will require higher-precision data.

\section{ACKNOWLEDGMENTS}

The authors acknowledge the funding of the German Federal Ministry of Education and Research (BMBF) within the NUSTAR R\&D project (Grant no. 05P15RDFN1) and the German Research Foundation (DFG) within the CRC 1245. This work was supported by U.S. DOE under contract No. DE-AC02-05CH11231 (LBNL, GRETINA), DE-AC0206CH11357 (ANL) and DE-AC52-07NA27344 (LLNL), and grants No. DE-FG02-97ER41041 (UNC), and DEFG02-97ER41033 (TUNL) and by BgNSF under grant DN08/23/2016. This research used resources of ANL's ATLAS facility, which is a DOE Office of Science User Facility. We also thank the operators at the ANL ATLASfacility for providing the beam.
[1] A. L. Goodman, Nuclear Physics A, 287, 1 (1977).

[2] P. Federman and S. Pittel, Physics Letters B 69, 385 (1977).

[3] N. Zeldes, T. Dumitrescu, and H. Köhler, Nuclear Physics A 399, 11 (1983).

[4] B. H. Wildenthal and W. Chung, Phys. Rev. C 22, 2260 (1980).

[5] A. Poves and J. Retamosa, Physics Letters B 184, 311 (1987).

[6] E. K. Warburton et al., Phys. Rev. C 41, 1147 (1990).

[7] T. Otsuka et al., Phys. Rev. Lett. 104, 012501 (2010).

[8] C. J. Prokop et al., Phys. Rev. C 92, 061302(R) (2015).

[9] C. Santamaria et al., Phys. Rev. Lett. 115, 192501 (2015).

[10] D. Warner, Nature 420, 614 (2002).

[11] R. F. Casten and N. V. Zamfir, Phys. Rev. Lett. 85, 3584 (2000).

[12] F. Iachello, Phys. Rev. Lett. 85, 3580 (2000).

[13] V. Werner et al., Phys. Rev. C 78, 051303 (2008).

[14] T. Togashi et al., Phys. Rev. Lett. 117, 172502 (2016).

[15] C. Kremer et al., Phys. Rev. Lett. 117, 172503 (2016).

[16] H. T. Fortune, Phys. Rev. C 95, 054313 (2017).
[17] A. Chakraborty et al., Phys. Rev. Lett. 110, 022504 (2013).

[18] S. Ansari et al., Phys. Rev. C 96, 054323 (2017).

[19] G. Savard et al., Hyperfine Interactions 199, 301 (2011).

[20] G. Savard et al., Nucl. Instr. and Meth. in Phys. Res. B 376, 246 (2016).

[21] R. C. Pardo, G. Savard, and R. V. F. Janssens, Nuclear Physics News 26, 5 (2016).

[22] C. Wu et al., Nucl. Instr. and Meth. in Phys. Res. A 814, 6 (2016).

[23] D. Cline, Annual Review of Nuclear and Particle Science 36, 683 (1986).

[24] S. Paschalis et al., Nucl. Instr. and Meth. in Phys. Res. A 709, 44 (2013).

[25] B. Pritychenko et al., Nucl. Instr. and Meth. in Phys. Res. A 640, 213 (2011).

[26] B. Singh and Z. Hu, Nuclear Data Sheets for A=98, Nucl. Data Sheets 98, 335 (2003).

[27] T. England and B. Rider, Evaluation and Compilation of Fission Yields, ENDF-349, LA-UR-94-3106 (1994).

[28] G. Savard and R. Pardo, Proposal for the 252Cf source upgrade to the ATLAS facility, Technical report, ANL 
(2005).

[29] H. Ower et al., Computer program CLX, PhD Thesis Goethe university of Frankfurt am Main (original 1980).

[30] K. Sieja et al., Phys. Rev. C 79, 064310 (2009).

[31] G. J. Kumbartzki et al., Phys. Rev. C 85, 044322 (2012).

[32] K. Nomura, R. Rodríguez-Guzmán, and L. M. Robledo,
Phys. Rev. C 94, 044314 (2016).

[33] H. Abusara and S. Ahmad, Phys. Rev. C 96, 064303 (2017).

[34] C. Fransen et al., Phys. Rev. C 71, 054304 (2005).

[35] A. Lisetskiy et al., Nuclear Physics A 677, 100 (2000). 